\title{
EL HILEMORFISMO EN EVOLUCIÓN. UNA APROXIMACIÓN MORAL A LA RELACIÓN ENTRE EL CUERPO Y EL ALMA EN ARISTÓTELES
}

\author{
Diego Sebastián Garrocho Salcedo* \\ doi:10.11144/Javeriana.uph33-67.heca
}

\begin{abstract}
RESUMEN
El presente artículo trata de reconstruir la eventual continuidad doctrinal en el tratamiento aristotélico entre el alma y el cuerpo. Primeramente, trataremos de problematizar las clásicas dicotomías en las que se ha intentado encajar la teoría hilemórfica con vistas a demostrar la imposibilidad de defender bien sea un dualismo o bien un monismo que resuma, con exactitud suficiente, el tratamiento aristotélico del alma. Finalmente, y a la luz de los planteamientos definitivos que parecen recogerse en De Anima, abordaremos un análisis comparado entre este tratado tardío y el examen de la relación entre el alma y el cuerpo contenido en los textos morales del Estagirita. Palabras clave: Aristóteles; cuerpo; alma; pasiones; hilemorfismo
\end{abstract}

* Universidad Autónoma de Madrid, Madrid, España.

Correo electrónico: diego.garrocho@uam.es

Para citar este artículo: GARROCHO SALCEDO, D.S. (2016). El hilemorfismo en evolución. Una aproximación moral a la relación entre el cuerpo y el alma en Aristóteles. Universitas Philosophica, 33(67), pp. 165-181. ISSN 0120-5323, ISSN en línea: 2346-2426, doi:10.11144/Javeriana.uph33-67.heca 


\title{
HYLOMORFISM IN EVOLUTION. A MORAL APPROACH TO THE RELATION BETWEEN BODY AND SOUL IN ARISTOTLE
}

\author{
Diego Sebastián Garrocho Salcedo
}

\begin{abstract}
This paper aims to restore the doctrinal continuity on the basis of the Aristotelian analysis of the relation between body and soul. First, we attempt to bring into question some classical dichotomies used to summarize the hylomorphic theory. We shall also try to demonstrate the impossibility of characterizing this theory as monism rather than dualism. Finally, in light of the doctrine that seems to be exposed in De Ani$m a$, we will do a comparative analysis between this final period of the Aristotelian thought and the thesis contained within his moral texts.

Key Words: Aristotle; body; soul; passions; hylomorfism
\end{abstract}


Pocas Conjunciones convocan, en la historia de la filosofía, más conflictos o pares de contrarios de los que podría alumbrar la oposición de aquello que los griegos distinguieron como $\sigma \tilde{\omega} \mu \alpha$ y $\psi v \chi \dot{\eta}$, y que hoy, tal vez impropiamente, infringiendo las reglas de la lealtad imposible que acecha a toda traducción, tratamos de resumir con nuestros conceptos de "cuerpo" y "alma". Esta querella anciana, tan antigua como el nacimiento mismo de la filosofía, es un binomio que resume consigo enfrentamientos fecundos sobre los que se reúnen tradiciones, disputas y relatos que hoy todavía reverberan en la inquietud de cualquier hombre. Eternidad y mortalidad, naturaleza y cultura, visible e invisible, carne y espíritu y, hasta incluso, hechos e interpretaciones, etc., son, si se quiere, inflexiones o declinaciones de la vieja disputa que trataba de resolver la congénita y constitutiva dualidad del hombre.

Ante esto, quizá merezca la pena atender las palabras de un viejo adagio griego, de Hesíodo o algún otro sabio anterior, que advertía que el principio es más

1 El presente artículo tratará de examinar la vigencia, utilidad y rendimiento hermenéutico del tratamiento del problema mente-cuerpo en el pensamiento aristotélico. Desde las últimas décadas del siglo XX (Thiebaut, 2004) hasta nuestros días, la filosofía de Aristóteles ha gozado de una renovada visibilidad aunque esta querencia haya acusado, en no pocas ocasiones, algunos excesos al intentar ejercer una defensa tan partidista como desmedida del legado conceptual del pensador de Estagira. Lejos de sumarnos a este "fundamentalismo aristotélico" que describiera R. Wallach (1992), nuestra propuesta se mostraría mucho más próxima a la actitud adelantada por el profesor Alejandro Vigo (2006) en relación, por ejemplo, con la física. No se trataría, pues, de evaluar el encaje de la filosofía aristotélica en las categorías dominantes de la psicología actual, sino de problematizar esos estándares dicotómicos -y todo lo que, en su resumida concepción de la realidad, desechan- sirviéndonos, precisamente, de una propuesta históricamente ajena al contexto de gestación del enfrentamiento entre el paradigma dualista y el monista hasta forzar su colapso. En otras palabras, con este artículo intentaremos capitalizar algunas aportaciones de la ética clásica con vistas a activar un nuevo marco comprensivo en lo que concierne a la relación mente-cuerpo. Para ello, proponemos revisar la coherencia del planteamiento aristotélico en relación a este problema atendiendo, fundamentalmente, al carácter evolutivo de su obra. Seguidamente, constataremos las dificultades en las que se han encontrado los estudiosos - preferentemente de la tradición anglosajona- al intentar ubicar, en una tentativa inidónea, la psicología aristotélica en alguno de los dos extremos del debate. Para el desarrollo de esta propuesta nos serviremos, prioritariamente, del carácter liminar del tratamiento de las pasiones en la psicología aristotélica como caso límite que resultaría inasible para las descripciones habituales en términos de dualismo o monismo. 
de la mitad del todo. Vayamos pues, a un principio, que como tantos, habrá de expresarse en forma de palabras, en este caso las de Aristóteles, padre de tantas ciencias y, según Kant, también clausura dede la lógica; discípulo de aquel Platón del que los manuales nos dijeron que siguió con puntual celo las enseñanzas de aquella secta ilustre: el orfismo, para escindir de una vez por todas la unidad del hombre hasta hacer del cuerpo y el alma dos realidades separadas. Al respecto, con demasiada frecuencia olvidamos que ya Olimpiodoro nos advirtió que aquella inspiración, que aquella respiración órfica del padre de todas las academias, no fue fiel al espíritu de la secta porque Platón intentó adaptar aquel legado para el provecho de su propia doctrina.

Como de herencias hablamos, al igual que ocurriera con la partición del alma, en la filosofía aristotélica encontramos una clara inspiración platónica acerca de la relación alma-cuerpo. De Platón recordamos la célebre caracterización del cuerpo como cárcel o tumba del alma², por lo que el de Atenas ha sido caracterizado tradicionalmente como el filósofo inaugural del dualismo. La inmortalidad del alma y la distribución jerárquica entre las distintas capacidades del hombre parecen garantizar, como sostuvo S. Broadie (2001), que es Platón quien inicia la tradición dualista y quien identifica por primera vez la identidad subjetiva con la dimensión anímica y no con la corporal. En el caso del discípulo, Aristóteles, la complejidad de su descripción del alma y el cuerpo han impedido concluir de un modo tajante su adscripción a un modelo ortodoxamente monista o dualista. Las vías de acceso al problema, como casi siempre, podrían ser varias, pero quisiera respetar la proporcionalidad para exigirnos, como dice el refrán, grandes problemas para grandes soluciones. A este respecto, de cuantos escenarios pudieran si no arrojar luz sí al menos orientar las sombras, una clave pertinente para interpretar la relación entre el cuerpo y el alma en Aristóteles habrían de ser, deberían ser, esos acontecimientos liminales de los que nunca sabemos ubicar su arraigo. Se trata, claro es, del único elemento verdaderamente motivador de cualquier vida: las pasiones $(\pi \dot{\alpha} \theta \eta)$.

Las pasiones son, pues, el referente de aquello que simbolizan las palabras. Así alcanzó a decirlo en un texto tan alejado, en principio, de cualquier celo ético o político en el que con paso exacto Aristóteles subrayó que las palabras ( $\gamma$ pa $\phi \dot{j} \mu \varepsilon v \alpha)$ 
simbolizan nuestra voz $(\phi \omega \nu \dot{\eta})$, y esta voz habría de simbolizar y representar las pasiones del alma. No es Descartes quien lo dice, sino el discípulo de Platón quien detrás de ese significativo desliz en un texto logicista, el Peri Hermeneias, no dejó de recordarnos en todos sus tratados que las pasiones, de ser, y son, de qué manera, habrán de ser siempre pasiones del alma: $\pi \dot{\alpha} \theta \eta \tau \tilde{\eta} s \psi v \chi \tilde{\eta} \varsigma^{3}$.

El hecho de Que Aristóteles caracterizara a las pasiones como acontecimientos específicamente psíquicos o anímicos no ha impedido que con respecto a la relación entre el alma y el cuerpo haya existido -y aún hoy se prolongue- una vivísima disputa entre los estudiosos. El primer obstáculo al que debe enfrentarse toda interpretación del alma en Aristóteles es a la aparente falta de univocidad y consistencia entre sus distintos tratados, una irregularidad fruto, quizá, de las venturas biográficas que asaltaron la vida del estagirita y de la posterior forma en la que se nos legaron sus escritos. Una explicación razonada de esta falta de coherencia la brindaron Nuyens y Ross (1948), quienes recurriendo a la hipótesis evolutiva del pensamiento Aristotélico trazaron una paulatina transformación de su psicología. Esta partiría del Eudemo - de clara inspiración platónica- y desembocaría, ya en el período de madurez, en el hilemorfismo expuesto en De Anima, siendo esta, por ello, su doctrina más genuina y elaborada. Más adelante evaluaremos la aceptación contemporánea de esta hipótesis pero, por lo pronto, merece la pena destacar que, incluso en referencia exclusiva al DA, no parece existir un consenso definitivo con respecto a la relación entre el alma y el cuerpo.

Jonathan Barnes (1971-1972) evidenció en los años sesenta el modo en que la psicología aristotélica había generado tanto lecturas materialistas como alternativas decididamente dualistas; poco tiempo después, Richard Sorabji (1974) capituló estas posiciones distintas y terminó por arrojar una serie de

3 Al respecto, véase: Int. I; DA 402a 9; 403a 3; 403 b17 \& Rhet.1354a17. En EN (1105b19) las pasiones se reconocen como aquello que ocurre en el alma junto con las facultades y las disposicio-

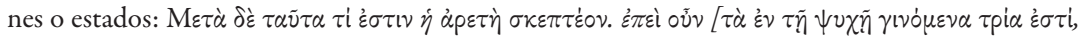

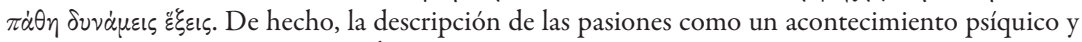
anímico es una constante en las Éticas. De aquí en lo que sigue, las referencias a EN seguirán la traducción de Julio Pallí Bonet (2003). 
conclusiones propias a este respecto. Como tantas veces, vemos, por tanto, que la relación entre el alma y el cuerpo en Aristóteles dista mucho de ser clara. Autores como Thomas Slakey privilegiaron la interpretación fisicalista; otros como Wallace Matson insistieron, no sin asumir ciertas generalizaciones simplistas, que la identificación entre cuerpo y alma resultaba obvia para la filosofía griega. En el extremo dualista, Sorabji situaba al propio Barnes y a disputas más contemporáneas como la celebrada entre M. Nussbaum, H. Putnam y M.F. Burneyat ${ }^{4}$, quienes demuestran que el hilemorfismo aún admite tanto interpretaciones dualistas como lecturas, si no enteramente físicalistas, sí cercanas al materialismo. En nuestra lengua, un análisis más rico en matices como el que elabora Marcelo Boeri (2007) sobre Barnes, podría dar cuenta de esta complejidad.

Gran parte del debate entre estos intérpretes se centra en el modo en que Aristóteles describe la percepción ( $\alpha \grave{i} \sigma \eta \eta \sigma \varsigma)$, aunque la mayoría de las razones y argumentos que ofrecen pueden hacerse extensivos también a las pasiones. En principio, existen buenas razones en una y otra dirección. Partiendo de la hipótesis monista cabe destacar que no son pocas las razones que asisten a la interpretación fisicalista de la psicología aristotélica. Las razones fundamentales que justifican tal hipótesis se concentran en el Libro II de DA, lugar en el que se describe el alma como $\dot{\varepsilon} \tau \varepsilon \lambda \dot{\varepsilon} \chi \varepsilon \varepsilon \alpha$, esto es, como acto del cuerpo. Esta afirmación convive con una comprensión preliminar que interpretada cabalmente dificulta su eventual validez e incluso, diríamos, se autoderrota. Conforme a la descripción aristotélica "la materia es potencia mientras que la forma es acto o entele-

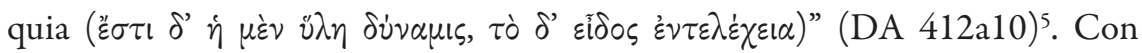
respecto a la relación entre el alma y el cuerpo, Aristóteles identifica el alma, precisamente, como el elemento distintivo de los organismos que tienen vida, es decir, es la $\psi v \chi \dot{\eta}$ lo que hace que un determinado cuerpo se distinga efectivamente como un cuerpo animado ( $\varepsilon \mu \psi v \times o v)$, esto es, dotado de vida ${ }^{6}$. En otras palabras:

4 Burneyat critica el funcionalismo de Nussbaum y Putnam en su texto de 2009 titulado "Is an Aristotelian Philosophy of Mind still Credible". Su crítica obtiene respuesta por parte de estos autores en "Changing Aristotle's Mind".

5 De aquí en lo que sigue, las citas a DA se acogerán a la traducción de Tomás Calvo (1978).

6 Por ello el alma en Aristóteles no es un elemento distintivo del hombre sino que es común a todos los seres vivos aunque admita niveles de complejidad. 
para Aristóteles "el alma es aquello por lo que vivimos" (DA 414a13). Esta caracterización justificaría una interpretación no substancialista del alma puesto que la substancia en este caso no sería el alma, sino el ser animado que resulta de la composición de la materia y la forma. Por tanto, en esta distribución existe una ordenación que identifica cuerpo y materia - distinguido por ser pura potenciay el alma, que constituye la forma o $\dot{\varepsilon} \tau \tau \varepsilon \lambda \dot{\varepsilon} \chi \varepsilon\llcorner\alpha$ del cuerpo animado. Barnes señala y advierte en su diagnóstico de la fragilidad de la interpretación monista, ya que con indudable rigor lógico una interpretación no sustancialista ${ }^{7}$ del alma no entraña necesariamente un fisicalismo ${ }^{8}$. Esto es, que el alma no sea una sustancia no significa que no sea nada. De hecho, podríamos añadir a su deducción lógica que no solo el hilemorfismo no entraña el no-sustancialismo del alma, sino que también comprometería la sustancialización del cuerpo; por lo que un fisicalismo fundamentado en la teoría hilemórfica no solo estaría injustificado sino que, incluso desde el nombre, me atrevería a decir, se demostraría contradictorio. Con todo, el hilemorfismo no solo compromete la sustancialización del alma, sino que la materia, en tanto que elemento constitutivo de la sustancia compuesta resultante -el cuerpo animado-, tampoco puede ser considerada como una sustancia independiente.

EL MONISMO FISICALISTA CUENTA con otros argumentos razonables a su favor. Así, por ejemplo, existen numerosas referencias en la obra de Aristóteles que parecen adelantar la intervención del cuerpo en procesos que, en principio, podrían reconocerse como puramente psíquicos. Un ejemplo de ello lo encontramos en Retórica, donde se establece que algunos procesos pasionales son debidos a una mera disposición corporal. Tal es el caso de la valentía del hombre anciano, la cual se ve mitigada en razón de su frialdad (opuesta al calor de los jóvenes) ${ }^{9}$.

7 La sustancialización del alma y sus partes ya suscitó un intenso debate en la recepción medieval de Aristóteles. Un sumario detallado y vinculado a la unidad subjetiva lo encontramos en Martin \& Barresi, 2006, p. 94.

8 Véase: Martin \& Barresi, 2006, p. 103.

9 Ret. 1389b30. Seguiremos siempre la traducción de Quintín Racionero (1990). 
También en DA se afirma que es la agitación del corazón la que ordena la persecución del placer (432b30) y que, precisamente, encolerizarse o atemorizarse son pasiones que consisten en un determinado movimiento ( zón (DA 408b7). Es más, en el Libro I de este tratado Aristóteles detalla cómo el físico describiría las pasiones como la ebullición de la sangre o del elemento caliente alrededor del corazón (DA 403b1), y parece adelantar, aunque con un estilo un tanto confuso, los distintos métodos desde los cuales cabría investigar las pasiones: o bien atendiendo a la definición, como haría el metafísico ${ }^{10}$; o bien atendiendo a la materia, como sería el caso referido del físico. Sin embargo, Aristóteles concluye el pasaje subrayando el hecho de que las pasiones del alma son inseparables de la materia natural de los animales. Recordemos, pese a todo, que la inseparabilidad nunca fue identidad. Con todo, esta última referencia constituye una razón de peso a favor de la interpretación fisicalista de Aristóteles, puesto que no simplemente predica la materialidad o corporalidad de las pasiones, sino que emplea de nuevo la expresión $\tau \dot{\alpha} \pi \dot{\alpha} \theta \eta \tau \tilde{\eta} \varsigma \psi v \chi \tilde{\eta} s$. Es decir, son precisamente las pasiones del alma las que acusan una naturaleza corporal insinuando con resumir en la condición corpórea las actividades propias del alma. De cuantas citas pudieran apoyar esta posición, una destaca por su manifiesta rotundidad. Así dice Aristóteles:

En la mayoría de los casos se puede observar cómo el alma no hace ni padece nada sin el cuerpo, por ejemplo, encolerizarse, envalentonarse, apetecer, sen-

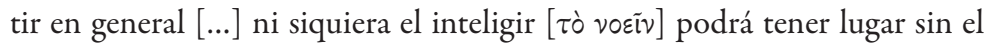
cuerpo si es que se trata de un cierto tipo de imaginación. (DA 403a5-7)

Esta advertencia, a pesar de su contundencia, no exige más que interpretar el cuerpo como una condición necesaria, pero habríamos de guardarnos de concluir que la dimensión somática es también condición suficiente. Con respecto a las pasiones, en sentido estricto, Aristóteles da un paso más. Su descripción fisicalista es constante y en distintos tratados adelanta la posibilidad de explicar los procesos pasionales como alteraciones fisiológicas. Jonathan Barnes (1971-1972, p. 108) insiste, y creemos que justificadamente, en el hecho de que por más que se requiera la intervención de un soporte físico o corporal las pasiones no pueden

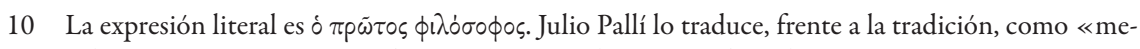
tafísico», a partir de la identificación de la metafísica como filosofía primera. 
identificarse con un proceso puramente material. De nuevo, el decir de Aristóteles parece claro a este respecto cuando afirma que "están en lo cierto cuantos opinan que el alma ni se da sin un cuerpo ni es en sí misma un cuerpo" (DA 403a20). Así, diremos, la corporalidad de las pasiones -y de tantos otros procesos psíquicos- sería una condición necesaria pero no suficiente. Ni siquiera se trataría de una característica esencial puesto que, respetando la doctrina hilemórfica, lo que determina la esencia del cuerpo no es la mera ordenación o el acontecimiento de ciertos procesos físicos, sino su forma o $\dot{\varepsilon} v \tau \varepsilon \lambda \dot{\varepsilon} \chi \varepsilon 1 \alpha$ : esto es, la $\psi v \chi \dot{\eta}$.

La crítica de Barnes a las interpretaciones fisicalistas parece razonablemente justificada y, sobre todo, con lo que acabamos de analizar, existen suficientes referencias textuales en Aristóteles como para distinguir los procesos anímicos de su pura dimensión corporal. En esta dirección abundará Richard Sorabji, quien completa la crítica de Barnes e incluso matiza el riesgo de abordar el examen de la psicología aristotélica a partir de categorías extemporáneas y ajenas a su contexto filosófico. De este modo, cifrar el debate en términos de monismo o dualismo no sería más que un anacronismo que lejos de arrojar luz sobre el problema favorecería la aceptación de conclusiones sesgadas e imprecisas. Por ello, siguiendo la advertencia de Sorabji, consideramos que la relación cuerpo-mente en Aristóteles no puede resolverse a partir de una dialéctica de extremos dualistas o fisicalistas sino que, con vistas a examinar el estatuto moral de las pasiones y su relación con el cuerpo, debemos analizar las singularidades específicas de la doctrina psicológica aristotélica. Para Sorabji, de hecho, la relación alma-cuerpo en Aristóteles no podría describirse como un dualismo al estilo cartesiano sino que, vagamente apunta el autor, debemos distinguirla como una concepción sui generis.

Esta interpretación es solo en parte semejante a la de Barnes puesto que preliminarmente también parte del carácter no-sustancialista del alma aristotélica, es decir: el alma se caracteriza por ser un agregado de capacidades o facultades y no por ser una sustancia separada e independiente (Sorabji, 1974, p. 64). Sorabji se sirve de la explicación causal para evidenciar el modo en que los procesos psíquicos trascienden el nivel fisiológico, por más que la concurrencia de la dimensión corpórea se demuestre imprescindible. Esta imposibilidad se muestra con especial claridad en una metáfora que se esboza en DA, pero que aparecerá 
detallada con toda significatividad en el Libro H de Metafisica ${ }^{11}$. En torno a la definición de una casa, por ejemplo, Aristóteles señala que aquellos que la definen como un conjunto de piedras, maderas y ladrillos, describen la casa en potencia ( $\delta \dot{v} v \alpha \mu \iota \zeta)$. Sin embargo, el acto ( $\dot{v} \dot{\varepsilon} \rho \gamma \varepsilon ı)$ de la casa consiste en ser refugio del calor y la lluvia, y el compuesto de ambas funciones daría como resultado la tercera sustancia compuesta de materia y forma. Hay por tanto, así en la materia como en la forma -tanto en el cuerpo como en el alma-, algo análogo a la sustancia pero que no puede ser, como señalaron Sorabji y Barnes, considerado como tal. De ahí que la sustancia no pueda reducirse ni a materia ni a forma, sino que se distingue como ov̉ $\sigma i \alpha$ en su cualidad sintética o compuesta. Subsumiendo esta explicación general al caso del alma y el cuerpo, diremos, que ni la explicación corpórea ni la pura $\varepsilon \dot{v} \tau \varepsilon \lambda \varepsilon \dot{\varepsilon} \chi \varepsilon \dot{\alpha}$ del alma pueden dar cumplida del compuesto del cuerpo animado ni sus procesos.

4.

A LA LUZ DE estos ARgUMENTOS PARECE SENSATO concluir que al menos en su período de madurez Aristóteles no puede ser interpretado como un autor fisicalista. El problema surge cuando tratamos de extrapolar estas conclusiones a su doctrina moral ya que, tanto evolutiva como metodológicamente ${ }^{12}$, las Éticas de Aristóteles no tendrían por qué guardar necesariamente una coherencia completa con la doctrina expuesta en DA. Sin embargo, de nuevo aparecen distintos motivos para sostener que las pasiones en los textos morales de Aristóteles no pueden definirse como puros procesos fisiológicos porque si atendemos a la estructura causal que desencadena un proceso pasional no parece que, por

11 Al respecto, véase: DA $403 a 26$ y ss; Met. H, $1043 a 12$ y ss. Para Met. nos remitimos a la traducción de Tomás Calvo (1994).

12 Sobre la metodología específica de la ética de Aristóteles, véase: Nussbaum, 1995, pp. 315-317 y Guariglia, 1992, pp. 21-25. Frente a Platón, Aristóteles elabora un discurso moral en el que las

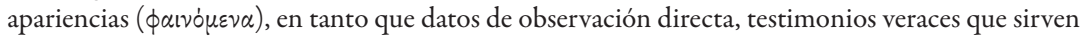
como garante de la investigación y sin los cuales no podría elaborarse ninguna teoría verdadera. La ideas de bien, virtud o justicia no dependerían de ninguna entidad suprasensible sino que adquirirían significación en la conducta propia del hombre bueno, virtuoso o justo. Parece, por tanto, una forma de «empirismo selectivo» en el que la naturaleza del ser humano se define no por la acción de los hombres sino por la conducta de aquellos individuos que podrían considerarse excelentes. 
ejemplo, la causa final pueda describirse en términos materiales. Así, en ocasiones podemos apetecer objetos reales, presentes y a los cuales tenemos acceso sensorial como ocurre en el caso del alimento. No obstante, Aristóteles también reconoce que podemos desear cosas imposibles (y hoy diríamos, sobre todo deseamos lo imposible) y algunas pasiones complejas, como por ejemplo el miedo en caso de ser infundado, que podrían tener una causa totalmente inmaterial. Los pasajes que reflejan una distinción entre el alma y el cuerpo son constantes a tal punto que tanto en Ética Nicomáquea como en Ética Eudemia se distingue

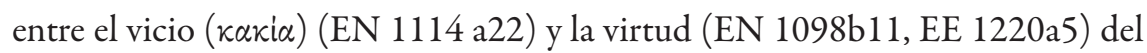
alma y los del cuerpo. De hecho, ahondando en esta distinción jerárquica, Aristóteles retoma la metáfora del maestro y establece una semejanza entre la relación de la ética y el alma y la medicina y el cuerpo (EN 1105b15).

Llegados a este punto, es necesario dar un paso más. Según la tesis aquí propuesta, tanto en los textos morales como en sus escritos de madurez, Aristóteles parece reconocer una doble naturaleza -anímica y somática- en el hombre. Tal condición se haría incluso extensiva a todos los seres vivos puesto que, como dijimos, la diferencia específica del cuerpo vivo es precisamente tener una psyché que lo anime. En el caso de $\mathrm{DA}^{13}$ la relación entre el cuerpo y el alma se resuelve a partir del esquema hilemórfico, por lo que la mutua dependencia ontológica y funcional parece garantizar una íntima cooperación entre la dimensión anímica y la corporal. A partir de la hipótesis evolutiva de Nuyens, suscrita también por intérpretes de la categoría de D. Ross o A. Gauthier y J.Y. Jolif, se impuso una interpretación que distinguió epocalmente las distintas fases de elaboración de la psicología aristotélica. Lo más relevante de esta teoría evolutiva es que restringía el paradigma hilemórfico a los escritos de madurez de Aristóteles. En esta distribución cronológica las Éticas quedarían ubicadas en un período de transición en las que la doctrina hilemórfica aún no habría sido elaborada. Poco o muy poco podríamos añadir a una disputa que, con mucho, excede nuestras posibilidades e instrumentos de análisis. Sin embargo, con respecto a la caracterización de la relación entre el alma y el cuerpo es razonable suscribir, a la vista de algunos de los pasajes

13 Ross (1995, p. 66) restringe el esquema hilemórfico a los Libros II y III de DA, negando su existencia en De Sensu y De Memoria. No así Hardie para quien el modelo hilemórfico se hace extensivo a todos los textos de madurez. 
referidos, una tesis cercana a lo que W.F.R. Hardie (1964) y I. Block (1961) sostuvieron hace tiempo, a saber: que si bien la evolución del pensamiento aristotélico puede ofrecer una cierta garantía en la ordenación cronológica de sus tratados, no existen razones definitivas que permitan concluir la existencia de dos doctrinas distintas e incompatibles entre sí sobre la relación entre el alma y el cuerpo.

Tanto Nuyens como Ross (1955) justifican la prioridad de Parva Naturalia con respecto al DA al considerar que el hilemorfismo - doctrina definitiva- sería incompatible con la doctrina expuesta en Parva Naturalia, que defiende el asentamiento del alma en un órgano singular. En De Sensu (436a7), las pasiones se describen específicamente como algo común al cuerpo y al alma "kovvd $\tau \tilde{\eta} \varsigma \tau \varepsilon$

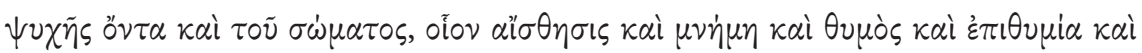

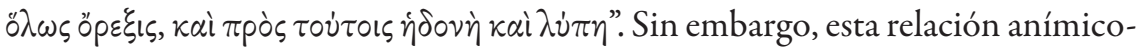
corporal no se describe como una unidad sintética o compuesta sino que, según señala Ross en su edición, que el alma se nutra especialmente de un órgano privilegiado como el corazón -el más caliente de todos (Sens. 438b30) - sería incompatible con la doctrina hilemórfica. Para Nuyens (1948, p. 253) ${ }^{14}$, igualmente, el asentamiento del alma en un órgano y la interpretación del alma como $\dot{\varepsilon} \nu \tau \varepsilon \lambda \dot{\varepsilon} \chi \varepsilon i \dot{\alpha}$ serían incompatibles. De este modo, siempre según Ross y Nuyens, las doctrinas expuestas en los textos anteriores ${ }^{15}$ a DA (tal es evidentemente el caso de EE y EN y, según su interpretación, también de Parva Naturalia) evidenciarían una suerte de dualismo más platónico en el que, casi cartesianamente, la sustancia anímica se aloja en un órgano privilegiado desde el cual se comanda el movimiento al conjunto del cuerpo. Estas dos tesis, dualista en el período de transición y heterodoxamente monista pero no fisicalista en DA, serían por tanto

14 Además, ver: Block, 1961, p. 50; Hardie, 1964, p. 67.

15 En la nota 8 del artículo de I. Block se destacan algunos de los pasajes en los que el corazón juega algún papel fundamental en el asentamiento del alma: en Partibus Animalium el corazón se describe como "la causa original de la vida" (678b 2), "el principio de la vida" (665a12), y "la parte central y rectora del cuerpo en la que se aloja la parte sensorial del alma y la fuente de la vida" (678b3). En Parva Naturalia, en De Somno (456a5), el corazón se describe como principio de la sensación y del movimiento; en De Juventute 469a7, se reitera "que el principio de la parte sensitiva y nutritiva del alma está en el corazón”; en 469b15-18, la vida de las distintas partes del cuerpo se aviva en el corazón. En De Respiratione 474b10-14, la vida del cuerpo entero depende del calor de la sangre. Citamos según las traducciones de la Biblioteca Clásica Gredos. 
incompatibles y demarcarían la evolución cronológica de la psicología aristotélica en dos niveles.

CABen VARIAs Respuestas a la Distinción de Nuyens y Ross. La primera es que en el DA aparecen también referencias al corazón como un órgano especialmente relevante en el desencadenamiento de procesos psíquicos, concretamente, con respecto al deseo. Así, en el Libro III. 10 (432b27) se señala que es la agitación del corazón la que se conmueve ante lo terrible, generando así la pasión del miedo $(\phi \dot{o} \beta \circ \varsigma)$. Sin embargo, para Ross esta referencia no constituiría un verdadero argumento ya que según su opinión la doctrina hilemórfica solo aparece reflejada en el Libro II y los ocho primeros capítulos del Libro III. Tampoco parece evidente que la consideración privilegiada del corazón entrañe una sustancialización ni del cuerpo ni del alma como sí ocurriera, por ejemplo, en el caso de Descartes y su célebre concepción de la glándula pineal. Bien es cierto que la definición de las pasiones en DA está referida a la dimensión corporal (403a16-19) mientras que en EN no se hace mención explícita al cuerpo (1105b19-23). Sin embargo, la separación entre cuerpo y alma no aparece reflejada en EN -inequívocamente escrito de transición- puesto que, como vimos, muchos procesos anímicos aparecen vinculados a alteraciones del cuerpo (incluso el hombre contemplativo requiere la salud del cuerpo para poder desarrollar su actividad teórica (EN 1178a37)). Igualmente, dado que la expresión "pasiones del alma" es frecuente en DA, como señalamos algunas páginas atrás, ni la corporalidad de las pasiones es exclusiva del período de madurez ni en el período de transición encontramos una descripción puramente psicológica del $\pi \dot{\alpha} \theta_{0}$. De este modo, a lo largo de la obra de Aristóteles encontramos una permanente coherencia al categorizar las pasiones como un proceso psicofísico en el que el extremo anímico y el extremo corporal aparecen estrechamente vinculados (EN 1178a17).

Finalicemos con dos argumentos más. En primer lugar, Hardie subraya que Aristóteles se refiere ya en EN (1178a17) al ser humano como un compuesto ( $\sigma \dot{v} \nu \theta \varepsilon \tau \circ v)$, con lo que destaca que las virtudes relacionadas con este compuesto serán también humanas $(\dot{\alpha} \nu \rho \omega \pi \iota k \alpha i)$. El hombre se describe, por tanto, como una unidad constituida por distintos elementos y no por dos sustancias de las 
cuales deba privilegiarse una. Sin embargo, algunas líneas antes Aristóteles señala que el hombre puede identificarse con su mejor parte y que un hombre es primariamente $(\mu \dot{\alpha} \lambda ı \tau \tau \alpha)$ dicha parte superior en poder y en dignidad (EN 1178a3). Esta afirmación parece destacar una parte de la sustancia compuesta, lo que cuestionaría la visión unitaria y coherente con el hilemorfismo. Hardie trata de resolver la paradoja remitiéndose a un pasaje anterior (EN 1177b27) en el que Aristóteles distingue al voũs -parte superior a la que se refería en la definición de hombre- como divina $(\theta \varepsilon i \tilde{\iota})$ y no humana. La síntesis no puede tratarse de una composición del elemento humano y el elemento divino puesto que el rasgo divino se distingue específicamente del compuesto (EN 1177b27). De hecho, el planteamiento de Aristóteles es claro puesto que señala que la parte superior del hombre es, precisamente, superior al compuesto ${ }^{16}$ (y en buena lógica, lo que es superior al compuesto no puede distinguirse como uno de sus componentes). Así, consideramos que la estrategia de Hardie a este respecto resulta débil puesto que trata de defender su compatibilidad con el hilemorfismo excluyendo el voṽ de la naturaleza del hombre. Es cierto que Aristóteles identifica al voús como algo divino con respecto del hombre, pero inmediatamente afirma que debemos hacer todo esfuerzo para vivir de acuerdo con lo más excelente que hay en noso-

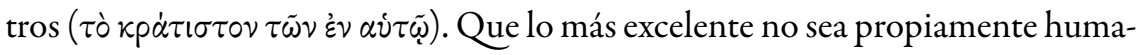
no sino divino no lo excluye, como defiende Hardie, sino que siguiendo al pie de la letra el decir de Aristóteles debemos vivir de acuerdo con esa parte excelente

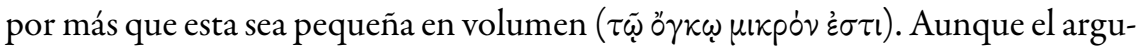
mento de Hardie no termine de parecernos concluyente creemos, pese a todo, que se trata de un detalle menor puesto que el problema de la integración del voũs en el seno del compuesto hilemórfico sería común al DA. Así, que en el Libro X de EN se opte por privilegiar un elemento como la parte más excelente no tiene por qué comprometer la coherencia de la psicología de este texto con la doctrina final del hilemorfismo. Además, siguiendo en esto a I. Block, puede que la solución definitiva se encuentre en la doctrina expuesta en Metafisica ( $\Delta$ 1035 b14-16 y ss) en la que, con mayor claridad, Aristóteles resuelve las distintas relaciones del todo y las partes en la sustancia compuesta.

16 Y por ello, no forma parte de los elementos que lo componen. 
En el Libro Z de Metafísica (1035b15 y ss.) Aristóteles aborda el problema de la relación entre las partes y el todo y atiende específicamente al carácter compuesto del cuerpo y el alma. En la relación entre ambos, en perfecto acuerdo con la doctrina hilemórfica, el alma se distingue como "especie y esencia del cuerpo"

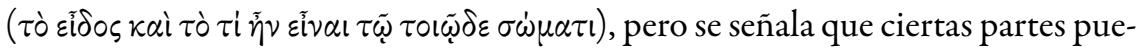
den ser anteriores al compuesto. Esta prioridad no sería simplemente cronológica sino también funcional y ontológicamente jerárquica, ya que sin ellas no podría darse ni el concepto ni la sustancia. En esta exposición Aristóteles destaca, por ejemplo, la relación del corazón y el alma, por lo que, como señala Block, el hilemorfismo no es incompatible en ningún caso con el privilegio de una parte del alma con respecto a las otras y al conjunto sintético resultante de la unión del alma y el cuerpo. Este pasaje se encuentra además refrendado por la hipótesis que adelanta en el Libro $\Delta$ (1113a4) en el que el de Estagira señala que el corazón o el cerebro son considerados por algunos autores como el principio desde el cual un animal se constituye como tal. Block señala además otro pasaje del mismo tratado en el que Aristóteles parece ahondar en este argumento ya que, en el mismo Libro $\Delta$, en referencia a la mutilación, Aristóteles distingue la jerarquía de algunas partes del cuerpo sobre otras.

A la vista de los distintos argumentos expuestos hasta acá, es suficientemente justificado sostener, por tanto, que la doctrina psicológica aristotélica guarda una razonable coherencia interna desde las Éticas a los tratados, en los que se expone explícitamente la doctrina del hilemorfismo. Con ello no pretendemos defender que el hilemorfismo estuviera ya presente en los escritos de transición ni tan siquiera que en, por ejemplo en EN, Aristóteles concibiera tácitamente la relación del alma y el cuerpo a partir del esquema hilemórfico. Los pasajes y argumentos que hemos rastreado no pretenden forzar una interpretación continua, pero sí mantener una cierta coherencia con respecto a la relación del alma y el cuerpo en la psicología aristotélica trazando, por cierto, una nada inocente continuidad entre sus presupuestos epistemológicos, biológicos y morales. Así, sostenemos que una interpretación ajustada evitaría, como ya dijimos, cualquier extremo que tratara de resumir la psicología aristotélica en esquemas monistas o dualistas.

Las pasiones -como también la percepción o incluso la intelección- evidencian un arraigo innegablemente corporal y, siguiendo el propio decir de Aristóteles, desde la perspectiva del físico podrían describirse como puras alteraciones 
fisiológicas, pero desde el punto de vista del metafísico, acaso nosotros, deberíamos siempre exigir una definición en la que el ser, esto es, el ser del alma, pueda decirse también de otra manera. Sin embargo, la reiterada descripción de las pasiones como acontecimientos anímicos en EN y la solución final del hilemorfismo nos llevan a pensar que en Aristóteles el cuerpo y el alma aparecen descritos como dos niveles que cooperan y se influyen íntimamente. Por eso, la pura descripción fisiológica o un animismo sin corporalidad dejarían de dar cuenta del modo en que Aristóteles describe las pasiones, lo que es tanto como decir el modo en que concibe al ser humano. Sumando, por tanto, estas tesis parciales, es justificado afirmar que en Aristóteles se adelanta una primera unidad de las partes del alma entre sí y una síntesis entre las funciones del cuerpo y las específicamente anímicas sin que, desde una perspectiva filosófica, pueda darse cuenta de los procesos fisiológicos ni de los psicológicos de una manera aislada.

Referencias

Aristóteles. (1990). Retórica. (Trad. Q. Racionero). Madrid: Gredos.

Aristóteles. (1992). Investigación sobre los animale.s (Trad. J. Pallí Bonet). Madrid: Gredos.

Aristóteles. (2000). Partes de los animales - Marcha de los animales - Movimiento de los animales. (E. Jiménez). Madrid: Gredos.

Aristóteles. (1994). Metafísica. (Trad. T. Calvo). Madrid: Gredos.

Aristóteles. (2003). Ética Nicomáquea-Ética Eudemia. (Trad. J. Pallí Bonet). Madrid: Gredos.

Barnes, J. (1971-1972). Aristotle's Concept of Mind. Proceedings of the Aristotelian Society (New Series), 72, pp. 101-114.

Block, I. (1961). The Order of Aristotle's Psychological Writings. The American Journal of Philology, 82(1), pp. 50-77.

Boeri, M. (2007). Apariencia y realidad en el pensamiento griego. Buenos Aires: Colihue.

Broadie, S. (2001). Soul and Body in Plato and Descartes. Proceedings of the Aristotelian Society (New Series), 101, pp. 295-308. 
Burneyat, M.F. (1992). Is an Aristotelian Philosophy of Mind Still Credible. M.C. Nussbaum \& A.O. Rorty (Eds.), Essays on Aristotle's De Anima (pp. 18-29). Oxford: Clarendon.

Bywater, I. (Ed.). (1962 [1894]). Aristotelis Ethica Nicomachea. Oxford: Clarendon Press.

Guariglia, O. (1992). Ética y política según Aristóteles. Buenos Aires: CEAM.

Hardie, W.F.R. (1964). Aristote's Treatement of the Relation between the Soul and the Body. Philosophical Quarterly, 14(54), pp. 53-72.

Martin, R. \& Barresi, J. (2006). The Raise and the Fail of the Self. New York: Columbia University Press.

Nussbaum, M.C. (1995). La fragilidad del bien. Madrid: Visor.

Nussbaum, M.C. \& Putnam, H. (2002). Changing Aristotle's Mind. M.C. Nussbaum \& A.O. Rorty (Eds.), Essays on Aristotle's De Anima (pp. 30-60). Oxford: Clarendon.

Nuyens, F. (1948). Lévolution de la psychologie d'Aristote. Lovaina: ISF.

Platón. (1987). Crátilo. Diálogos II. (Trad. J.L. Calvo). Madrid: Gredos.

Platón. (1987). Gorgias. Diálogos II. (Trad. J. Calonge). Madrid: Gredos.

Platón. (1988). Fedro. Diálogos III. (Trad. E. Lledó Iñigo). Madrid: Gredos.

Ross, W.D. (Ed.). (1964 [1959]). Aristotelis ars rhetorica. Oxford: Clarendon Press.

Ross, W.D. (Ed.). (1967 [1961]). Aristotle, De Anima. Oxford: Clarendon Press.

Ross, W.D. (Ed.). (1970 [1924]). Aristotle's Metaphysics. Oxford: Clarendon Press.

Ross, W.D. (Ed.). (1955). Parvalia Naturalia. Oxford: Clarendon Press.

Sorabji, R.R.K. (1974). Body and Soul in Aristotle. Philosophy, 49(187), pp. 63-89.

Thiebaut, T. (2004). Neoaristotelismos. Concepciones de la ética. Madrid: TrottaCSIC.

Vigo, A. (2006). ¿Está obsoleta la Física de Aristóteles? Estudios públicos, 102, pp. 43-67.

Wallach, R. (1992). Contemporary Aristotelianism. Political Theory, 20(4), pp. 613-64. 